\title{
Refractory asthma in the UK: cross-sectional findings from a UK multicentre registry
}

\author{
Liam G Heaney, ${ }^{1}$ Chris E Brightling, ${ }^{2}$ Andrew Menzies-Gow, ${ }^{3}$ Michael Stevenson, ${ }^{4}$ \\ Rob M Niven, ${ }^{5}$ on behalf of the British Thoracic Society Difficult Asthma Network
}

${ }^{1}$ Centre for Infection and Immunity, Queen's University of Belfast, UK

${ }^{2}$ Department of Infection, Inflammation and Immunity, Institute for Lung Health, University of Leicester, UK ${ }^{3}$ Royal Brompton Hospital, London, UK

${ }^{4}$ School of Medicine, Dentistry and Biomedical Sciences, Queen's University Belfast, UK ${ }^{5}$ North West Lung Centre, University of Manchester, Manchester, UK

\section{Correspondence to} Dr Liam Heaney, Centre for Infection and Immunity, Queen's University of Belfast, Level 8, Belfast City Hospital, Lisburn Road, Belfast BT9 7AB, UK; I.heaney@qub.ac.uk

Received 22 February 2010 Accepted 25 May 2010

\section{ABSTRACT}

Introduction Refractory asthma represents a significant unmet clinical need where the evidence base for the assessment and therapeutic management is limited. The British Thoracic Society (BTS) Difficult Asthma Network has established an online National Registry to standardise specialist UK difficult asthma services and to facilitate research into the assessment and clinical management of difficult asthma.

Methods Data from 382 well characterised patients, who fulfilled the American Thoracic Society definition for refractory asthma attending four specialist UK centres-Royal Brompton Hospital, London, Glenfield Hospital, Leicester, University Hospital of South Manchester and Belfast City Hospital-were used to compare patient demographics, disease characteristics and healthcare utilisation.

Results Many demographic variables including gender, ethnicity and smoking prevalence were similar in UK centres and consistent with other published cohorts of refractory asthma. However, multiple demographic factors such as employment, family history, atopy prevalence, lung function, rates of hospital admission/ unscheduled healthcare visits and medication usage were different from published data and significantly different between UK centres. General linear modelling with unscheduled healthcare visits, rescue oral steroids and hospital admissions as dependent variables all identified a significant association with clinical centre; different associations were identified when centre was not included as a factor.

Conclusion Whilst there are similarities in UK patients with refractory asthma consistent with other comparable published cohorts, there are also differences, which may reflect different patient populations. These differences in important population characteristics were also identified within different UK specialist centres. Pooling multicentre data on subjects with refractory asthma may miss important differences and potentially confound attempts to phenotype this population.

\section{INTRODUCTION}

Patients with difficult asthma (persistent symptoms and/or frequent exacerbations despite treatment at step 4/5 of British Thoracic Society (BTS) management guidelines ${ }^{1}$ ) represent a significant unmet clinical need ${ }^{2}{ }^{3}$; however, the evidence base for the assessment and management of this group of patients is small. ${ }^{14}$

In 2006, the BTS Research Committee together with physicians with a specialist interest in difficult asthma established a National Registry for dedicated UK Difficult Asthma Services. The aims were to standardise specialist clinical services, to further define and characterise clinical phenotypes in subjects with well characterised severe asthma and to facilitate research into the assessment and clinical management of difficult asthma.

Observational studies have suggested that after detailed systematic evaluation, $\sim 50 \%$ of patients referred with difficult to control asthma do not have refractory disease, but have multiple other mechanisms for persistent symptoms ${ }^{5-7}$ The National Registry includes UK centres operating established dedicated multidisciplinary assessment protocols to ensure identification of patients with well characterised refractory asthma. The aim of this paper is to describe the clinical features of a well characterised UK refractory asthma population from the National Difficult Asthma Registry and compare patient groups from individual centres.

\section{METHODS}

There are currently seven UK dedicated Specialist Difficult Asthma Services submitting data to the UK Registry, but the data presented in this paper are from the four pilot UK centres-Royal Brompton Hospital, London, Glenfield Hospital, Leicester, University Hospital of South Manchester and Belfast City Hospital.

The Registry is hosted online by Dendrite Clinical Systems and admits password-protected anonymised data, after fully informed written consent; individual centre data can be downloaded locally by registered users for audit purposes.

Subjects were entered into the Registry in a nonselected manner, and centres were asked to have 100 subjects entered by a predefined deadline. The data in this manuscript were utilised as part of an initial service evaluation between clinical centres, and represent subjects, who after detailed assessment, fulfilled the American Thoracic Society (ATS) definition of refractory asthma. ${ }^{8}$

\section{Statistical analysis}

Anonymised data were analysed using Statistical Package for Social Sciences (SPSS, Chicago, Illinois, USA), Version 16. Between-centre comparisons were made using one-way analysis of variance (ANOVA) and Kruksal-Wallis testing, with posthoc comparisons using Bonferroni and Games-Howell comparisons, as appropriate. For categorical variables, comparisons were made using $\chi^{2}$ analysis with exact tests as appropriate. As multiple between-centre comparisons were made, statistical significance was taken as $p<0.01$ to minimise the number of results exhibiting type 1 
error. General linear modelling was used to generate models with the dependent variables unscheduled care visits, rescue oral steroids and hospital admissions (entered as the square root of the index variable to ensure residuals in the model were normally distributed). For intensive care unit (ICU) admission, only 71 of 376 cases had prior ICU admission, so a binary logistic regression model was used. In all modelling, final model selection was parsimonious, with accepted variables having statistical significance of $\mathrm{p}<0.05$.

\section{RESULTS}

Group data and between-centre comparisons are shown in tables $1-6$.

\section{Demographics}

There was a predominance of females, and more ethnic white Caucasians in all centres (table 1 ). In three of the centres $\sim 1 / 3$ of subjects were in full-time employment, but this was significantly higher in Leicester (58.2\%). Patients were less likely to record asthma as the reason for not working in Belfast, compared with Manchester and London, which had similar prevalences of full-time employment.

A family history of asthma was more common in Belfast and an atopic history highest in London (71.7\%); there were also significant between-centre differences in the recorded prevalence of perennial rhinitis and seasonal rhinitis (table 2). Subjects in Belfast and London were more likely to have had prior nasal surgery compared with Leicester and Manchester. Of note, few premenopausal women recorded significant premenstrual asthma worsening.

\section{Atopy}

The higher atopic prevalence in London was supported by allergen testing (table 3), with greater inhalant allergen sensitisation compared with Belfast (Leicester and Manchester use a clinically targeted allergen testing approach). The higher London atopic prevalence was not explained by younger 'age of asthma onset', because in subjects with asthma diagnosis $\geq 10$ years, allergic sensitisation remained higher (eg, house dust mite-positive subjects: Belfast, 18 of 59 (30.5\%) vs London, 38 of $41(92.7 \%))$.

\section{Healthcare utilisation}

In terms of healthcare utilisation, unscheduled visits (defined as general practitioner (GP)/hospital unscheduled contact) were higher in Belfast compared with other centres (table 4). Prereferral rescue steroid courses were lower in Manchester, although the hospital admission rate was highest in London, as was prior admission to an ICU.

\section{Medication}

The number of subjects taking maintenance oral steroids was highest in London, though the median dose was not different between centres (table 4). Inhaled steroid dose was significantly higher in London compared with other centres, and patients were more likely to be receiving theophyllines in London and leukotriene receptor antagonists in London and Belfast. There was a range of nebuliser usage, but again this was highest in London (66\%) and lowest in Leicester (24\%). Few patients were on anti-immunoglobulin $\mathrm{E}$ ( $\mathrm{IgE}$ ) treatment or steroid-sparing

Table 1 Patient demographic data

\begin{tabular}{|c|c|c|c|c|c|c|}
\hline & All (382) & Belfast (95) & Brompton (99) & Leicester (79) & Manchester (109) & \\
\hline Female (\%) & $241(63.1)$ & $56(58.9)$ & $63(63.6)$ & $45(57.8)$ & $77(70.6)$ & $p=0.259$ \\
\hline \multicolumn{7}{|l|}{ Race, n (\%) } \\
\hline White & $346(90.6)$ & $95(100)$ & $82(82.8)$ & $64(81.0)$ & $105(96.3)$ & $\mathrm{p}<0.001$ \\
\hline African & $10(2.6)$ & 0 & $7(7.1)$ & 0 & $3(2.8)$ & \\
\hline Unknown & $4(1.0)$ & 0 & $1(1.0)$ & 0 & $3(2.8)$ & \\
\hline $\begin{array}{l}\text { Age at first assessment at Difficult } \\
\text { Asthma Service (n) }\end{array}$ & $44.9 \pm 13.7(381)$ & $43.2 \pm 14.4(95)$ & $43.0 \pm 13.1$ (99) & $45.7 \pm 13.6(78)$ & $47.5 \pm 13.4(109)$ & $\mathrm{p}=0.055$ \\
\hline Height, $m(n)$ & $1.66 \pm 0.096(382)$ & $1.65 \pm 0.09(95)$ & $1.68 \pm 1.0(99)$ & $1.66 \pm 0.11(79)$ & $1.65 \pm 1.0(109)$ & $p=0.118$ \\
\hline Weight, kg (n) & $81.2 \pm 19.9(374)$ & $81.3 \pm 19.8(95)$ & $83.8 \pm 18.3(99)$ & $81.8 \pm 23.1(71)$ & $78.3 \pm 19.2(109)$ & $\mathrm{p}=0.254$ \\
\hline BMI (n) & $28(24.3-32.4)(374)$ & $28(25-34)(95)$ & $30(25-32)(99)$ & $28.4(24.4-34.5)(71)$ & $27(24-31)(109)$ & $\mathrm{p}=0.291$ \\
\hline \multicolumn{7}{|l|}{ Smoking status } \\
\hline Never & $233(61.0)$ & $57(60.0)$ & $69(69.7)$ & $50(63.3)$ & $57(52.3)$ & $p=0.182$ \\
\hline Ex-smoker (years since stopped) & $114(29.8)(8.97 \pm 8.15)$ & $27(28.4)(11.0 \pm 9.92)$ & $25(25.2)(9.64 \pm 6.72)$ & $24(30.4)(10.35 \pm 8.26)$ & $38(34.9)(5.55 \pm 6.35)$ & \\
\hline Asthma-related not working (\%) & $100(26.2)$ & $16(16.8)$ & $40(40.4)$ & $9(11.4)$ & $35(32.1)$ & \\
\hline Other cause for not working (\%) & $104(27.2)$ & $39(41.0)$ & $15(15.2)$ & $22(27.8)$ & $28(25.7)$ & \\
\hline Part-time working due to asthma (\%) & $12(3.1)$ & $0(0)$ & $10(10.1)$ & 0 & $2(1.8)$ & \\
\hline $\begin{array}{l}\text { Part-time working due to other cause } \\
(\%)\end{array}$ & $10(2.6)$ & $2(2.1)$ & $1(1.0)$ & $1(1.3)$ & $6(5.5)$ & \\
\hline Unknown & $5(1.3)$ & $4(4.2)$ & $0(0)$ & $1(1.3)$ & 0 & \\
\hline Work-related worsening of asthma (\%) & & & & & & $p=0.98$ \\
\hline Yes & $19(5.0)$ & $4(4.2)$ & $5(5.0)$ & $4(5.1)$ & $6(5.5)$ & \\
\hline No & $354(92.7)$ & $89(93.7)$ & $94(95.0)$ & $69(87.3)$ & $102(93.6)$ & \\
\hline Not recorded & $9(2.3)$ & $2(2.1)$ & 0 & $6(7.6)$ & $1(0.9)$ & \\
\hline
\end{tabular}

Group data (mean \pm SD or median (IOR)) for all subjects are presented in column 2 followed by data for individual centres. Between-centre comparisons for continuous variables were made using one-way analysis of variance or Kruksal-Wallis test (for posthoc comparisons, see text) and for categorical variables using $\chi^{2}$ exact testing.

Significance was taken as $\mathrm{p}<0.01$.

BMl, body mass index. 
Table 2 Medical history

\begin{tabular}{|c|c|c|c|c|c|c|}
\hline & All (382) & Belfast (95) & Brompton (99) & Leicester (79) & Manchester (109) & \\
\hline \multicolumn{7}{|l|}{ Family history of asthma (\%) } \\
\hline Yes & $193(50.5)$ & $70(73.7)$ & $44(44.4)$ & $33(41.8)$ & $46(42.2)$ & \multirow[t]{2}{*}{$\mathrm{p}<0.001$} \\
\hline No & $168(44.0)$ & $24(25.3)$ & $54(54.5)$ & $33(41.8)$ & $57(52.3)$ & \\
\hline \multicolumn{7}{|l|}{ Family history of asthma death (\%) } \\
\hline Yes & $12(3.1)$ & $5(5.3)$ & $3(3.0)$ & 0 & $4(3.7)$ & \multirow[t]{2}{*}{$\mathrm{p}=0.439$} \\
\hline No & $175(45.8)$ & $63(66.3)$ & $40(40.4)$ & $31(39.2)$ & $41(37.6)$ & \\
\hline Yes & $219(57.3)$ & $58(61.1)$ & $71(71.7)$ & $48(60.8)$ & $42(38.5)$ & \multirow[t]{3}{*}{$\mathrm{p}<0.001$} \\
\hline No & $159(41.6)$ & $37(38.9)$ & $28(28.3)$ & $31(39.2)$ & $63(57.8)$ & \\
\hline Missing & $4(1.1)$ & 0 & 0 & 0 & $4(3.7)$ & \\
\hline \multicolumn{7}{|l|}{ Perennial rhinitis (\%) } \\
\hline Yes & $111(29.0)$ & $44(46.3)$ & $35(35.4)$ & $17(21.5)$ & $15(13.8)$ & $\mathrm{p}<0.001$ \\
\hline No & $231(60.5)$ & $65(68.4)$ & $50(50.5)$ & $44(55.7)$ & $72(66.1)$ & \multirow{2}{*}{$\mathrm{p}<0.001$} \\
\hline Missing & $11(2.9)$ & $1(1.0)$ & $1(1.0)$ & 0 & $9(8.2)$ & \\
\hline \multicolumn{7}{|l|}{ Eczema (\%) } \\
\hline Yes & $103(27.0)$ & $27(28.4)$ & $28(28.3)$ & $24(30.4)$ & $24(22.0)$ & \multirow[t]{3}{*}{$\mathrm{p}=0.77$} \\
\hline No & $270(70.7)$ & $68(71.6)$ & $70(70.7)$ & $55(69.6)$ & $77(70.6)$ & \\
\hline Missing & $9(2.4)$ & 0 & $1(1.0)$ & 0 & $8(7.3)$ & \\
\hline \multicolumn{7}{|c|}{ Other atopic condition (\%) (food hypersensitvity, anaphylaxis, etc.) } \\
\hline Yes & $53(13.9)$ & $17(17.9)$ & $8(8.1)$ & $20(25.3)$ & $8(8.1)$ & \multirow[t]{3}{*}{$\mathrm{p}=0.002$} \\
\hline No & $320(83.8)$ & $78(82.1)$ & $90(90.9)$ & $59(74.7)$ & $93(84.7)$ & \\
\hline Missing & $9(2.4)$ & 0 & $1(1.0)$ & 0 & $8(7.2)$ & \\
\hline \multicolumn{7}{|l|}{ Prior nasal surgery (\%) } \\
\hline Yes & $55(14.4)$ & $20(21.1)$ & $20(20.2)$ & $8(10.1)$ & $7(6.4)$ & $\mathrm{p}=0.009$ \\
\hline No & $214(56.0)$ & $39(41.1)$ & $54(54.5)$ & $54(68.4)$ & $67(61.5)$ & \multirow{2}{*}{$\mathrm{p}<0.001$} \\
\hline Missing & $10(2.6)$ & 0 & $1(1.0)$ & $1(1.3)$ & $8(7.3)$ & \\
\hline Prior OGD (\%) & $17(3.7)$ & $6(6.3)$ & $5(5.0)$ & $4(5.1)$ & $2(1.8)$ & $\mathrm{p}=0.51$ \\
\hline Prior pH profile (abnormal profile) & $36(19)$ & $13(6)$ & $19(11)$ & $1(0)$ & $3(2)$ & $\mathrm{p}=0.62$ \\
\hline Significant catamennial asthma (\%) & 16 of 188 women (8.5) & 4 of $41(9.8)$ & 5 of $55(9.1)$ & 2 of $34(5.9)$ & 5 of $58(8.6)$ & $\mathrm{p}=0.939$ \\
\hline
\end{tabular}

Group data, $\mathrm{n}(\%)$ for all subjects, are presented in column 2 followed by data for individual centres. Between-centre comparisons were made using $\chi^{2}$ exact testing.

Significance was taken as $\mathbf{p}<0.01$.

OGD, oesophagogastroduodenoscopy.

medication at referral (three Manchester patients on omalizumab had started in a clinical trial before referral).

\section{Lung function}

Prebronchodilator forced expiratory volume in $1 \mathrm{~s}\left(\mathrm{FEV}_{1}\right)$ for all subjects and degree of airflow obstruction was similar in Manchester, London and Belfast; $\mathrm{FEV}_{1}$ was significantly higher and less obstructive in Leicester (table 5). In those subjects where a postbronchodilator study was available, again prebronchodilator spirometry was significantly better in Leicester compared with other centres, and postbronchodilator $\mathrm{FEV}_{1}$ was significantly better in Leicester compared with Belfast. Residual volume and total lung capacity were significantly greater in London compared with Belfast and Manchester, but $\mathrm{K}_{\mathrm{CO}}$ (carbon monoxide transfer coefficient) was normal and similar in all centres.
When lung function was compared between smoking groups, only $\mathrm{K}_{\mathrm{CO}}$ was different (never smokers 105.8 \pm 16.7 , ex-smokers 97.6 \pm 19.4 , current smokers 94.7 \pm 21.5 ); posthoc testing confirmed that $\mathrm{K}_{\mathrm{CO}}$ was lower in ex-smokers compared with never smokers $(p=0.020)$.

Inflammatory markers, immunoglobulins and bone densitometry The baseline blood eosinophil count was higher in Belfast compared with other centres, and posthoc testing confirmed that this difference was restricted to a difference between Belfast and London. Induced sputum, fractional exhaled nitric oxide (FeNO) and IgE showed no significant differences between centres (table 6).

\section{General linear modelling}

General linear models with dependent variables of unscheduled care visits, rescue oral steroids and hospital admission are shown in table 7. For prior ICU admission, binary logistic regression 
Table 3 Allergen testing

\begin{tabular}{|c|c|c|c|c|c|c|}
\hline & All (382) & Belfast (95) & Brompton (99) & Leicester (79) & Manchester (109) & \\
\hline \multicolumn{7}{|l|}{ Environmental allergen test (\%) } \\
\hline RAST only & $98(25.6)$ & $9(9.5)$ & $13(13.1)$ & $7(8.9)$ & $69(63.3)$ & \\
\hline SPT only & $36(9.4)$ & $5(5.2)$ & $4(4.0)$ & $18(22.8)$ & $9(8.2)$ & \\
\hline Not done & $22(5.8)$ & 0 & $3(3.0)$ & $4(5.1)$ & $15(13.8)$ & \\
\hline \multicolumn{7}{|l|}{ Domestic exposures (\%) pets } \\
\hline Yes (\%) & $116(30.4)$ & $27(28.4)$ & $20(20.2)$ & $29(36.7)$ & $40(36.7)$ & $p=0.026$ \\
\hline \multicolumn{7}{|l|}{ Aspergillus/fungi } \\
\hline Yes $(\%)$ & $18(4.7)$ & $1(1.0)$ & 0 & $4(5.1)$ & $13(11.9)$ & \multirow[t]{3}{*}{$\mathrm{p}<0.001$} \\
\hline No (\%) & $351(91.9)$ & $91(95.8)$ & $97(98.0)$ & 74 (93.7) & $89(81.7)$ & \\
\hline Not recorded (\%) & $13(3.4)$ & $3(3.2)$ & $2(2.0)$ & $1(1.3)$ & $7(6.4)$ & \\
\hline \multicolumn{7}{|l|}{ House dust mite $(\%)^{*}$} \\
\hline \multicolumn{7}{|l|}{ Cat $(\%)^{*}$} \\
\hline Positive & $159(41.6)$ & $29(30.5)$ & $77(77.8)$ & $18(22.8)$ & $35(32.1)$ & \multirow[t]{4}{*}{$\mathrm{p}<0.001$} \\
\hline Negative & $84(22.0)$ & $66(69.5)$ & $7(7.1)$ & $1(1.3)$ & $10(9.2)$ & \\
\hline Not done & $139(36.4)$ & 0 & $15(15.1)$ & $60(75.9)$ & $64(58.7)$ & \\
\hline$\%$ positivity for patients tested & $65.4 \%$ & $30.5 \%$ & $91.7 \%$ & $94.7 \%$ & $77.8 \%$ & \\
\hline \multicolumn{7}{|l|}{$\operatorname{Dog}(\%)^{*}$} \\
\hline Positive & $155(40.6)$ & $26(27.4)$ & $78(78.8)$ & $23(29.1)$ & $28(25.7)$ & \multirow[t]{4}{*}{$\mathrm{p}<0.001$} \\
\hline Negative & $95(24.9)$ & $68(71.6)$ & $7(7.1)$ & $1(1.3)$ & $19(17.4)$ & \\
\hline Not done & $132(34.5)$ & $1(1.0)$ & $14(14.1)$ & $55(69.6)$ & $62(56.9)$ & \\
\hline$\%$ positivity for patients tested & $62.0 \%$ & $27.7 \%$ & $91.8 \%$ & $95.8 \%$ & $59.6 \%$ & \\
\hline \multicolumn{7}{|l|}{ Mixed grasses $(\%)^{*}$} \\
\hline Positive & $149(39.0)$ & $31(32.6)$ & $77(77.8)$ & $26(32.9)$ & $15(13.8)$ & $\mathrm{p}<0.001$ \\
\hline
\end{tabular}

Group data, $\mathrm{n}(\%)$ for all subjects, are presented in column 2 followed by data for individual centres. Exposure to Aspergillus/fungi refers to these being apparent away from normal domestic areas (eg, windows, bathroom, etc.) Between-centre comparisons were made using $\chi^{2}$ exact analysis.

Significance was taken as $\mathrm{p}<0.01$.

RAST, radioallergosorbent; SPT, skin prick testing

*The data presented are for subjects with either a positive RAST or skin prick test.

modelling with ever ICU admission as dependent variable demonstrated significant associations with age of asthma diagnosis (OR 0.965 (95\% CI 0.948 to 0.984 ), p<0.001) and inhaled steroid dose per $100 \mu \mathrm{g}$ increase in beclomethasone dipropronate (BDP) equivalent (OR 1.029 (95\% CI, 1.003 to 1.055), $\mathrm{p}<0.05$ ).

\section{DISCUSSION}

This UK Difficult Asthma Network aims to standardise clinical assessment across specialist centres and use a Registry to facilitate phenotypic characterisation and research in patients with 'difficult asthma'. These specialist centres have established assessment protocols, ensuring subjects have well characterised refractory asthma. The numbers of subjects per centre provide further analytical strengths and, when data are consistent across three or four centres, we believe they are representative of the UK refractory asthma population. Between-centre differences are not explained by local referral patterns $(60-75 \%$ tertiary referrals, the remainder coming from primary/non-specialist physicians); however, this cannot be entirely excluded.
Three other multicentre studies have collated data on 'difficult asthma' previously. The TENOR study was a large-scale US observational study (4756 patients, 3489 adults) of difficult asthma ( $\geq 2$ unscheduled care visits or $\geq 2$ oral steroid bursts plus high dose inhaled steroids $)^{9} ; 51 \%$ were classified as severe asthma by the treating physician. The ENFUMOSA cross-sectional study (12 centres in nine European countries) recruited 163 patients with severe asthma ( $\geq 1$ exacerbation in the previous year despite $\geq 1200 \mu \mathrm{g}$ of BDP equivalent inhaled steroid). ${ }^{10}$ The SARP study included 204 subjects $\geq 12$ years, fulfilling the ATS definition of refractory asthma (9 US and 1 UK centre). ${ }^{11}$ In these studies, group data only are presented, whereas our data additionally allow comparison between individual UK centres, which has demonstrated important differences.

Our data confirm the usual female preponderance which remains unexplained, ${ }^{9-11}$ though catamennial asthma was not a common exacerbating factor.

Age at first assessment was similar between centres, though age at asthma diagnosis was significantly lower in the Brompton 
Table 4 Healthcare utilisation and medication

\begin{tabular}{|c|c|c|c|c|c|c|}
\hline & All (382) & Belfast (95) & Brompton (99) & Leicester (79) & Manchester (109) & \\
\hline $\begin{array}{l}\text { Unscheduled visits in } \\
\text { preceding } 12 \text { months (n) }\end{array}$ & $4(2-6)(372)$ & $5(2-9)(91)$ & $4(1-6)(99)$ & $4(2-6)(79)$ & $3(2-5)(103)$ & $p=0.007$ \\
\hline $\begin{array}{l}\text { Rescue steroid courses in } \\
\text { the previous year (n) }\end{array}$ & $4(2-6)(352)$ & $5(1-6)(84)$ & $5(2.75-7)(86)$ & $4(2-6)(79)$ & $2(0-4)(103)$ & $\mathrm{p}<0.001$ \\
\hline $\begin{array}{l}\text { Hospital admissions in } \\
\text { preceding } 12 \text { months ( } \mathrm{n} \text { ) }\end{array}$ & $0(0-2)(377)$ & $0(0-1)(93)$ & $1(0-3)(99)$ & $0(0-1)(79)$ & $0(0-1)(106)$ & $\mathrm{p}<0.001$ \\
\hline $\begin{array}{l}\text { Total number of ICU admission } \\
\text { (range) (n) }\end{array}$ & 0 Range $(0-11)(379)$ & 0 Range (0-4) (95) & 0 Range $(0-11)(99)$ & 0 Range $(0-1)(79)$ & 0 Range $(0-10)(106)$ & $\mathrm{p}=0.012$ \\
\hline Oral steroid dose $(\mathrm{mg})(\mathrm{n})$ & $15(10-20)(154)$ & $13(6-20)(31)$ & $15(10-20)(57)$ & $10(7.5-15)(30)$ & $15(10-30)(36)$ & $\mathrm{p}=0.021$ \\
\hline BDP equivalent dose $(\mu \mathrm{g})(\mathrm{n})$ & $2000(1000-2000)(362)$ & $1800(1000-2000) \mathrm{m}(93)$ & $2000(2000-2000)(94)$ & $2000(1600-2000)(78)$ & $1500(1000-2000)(97)$ & $\mathrm{p}<0.001$ \\
\hline SABA use per day & $6(4-8)(262)$ & $8(4-10)(31)$ & $4(6-8)(75)$ & $4(2-6)(62)$ & $8(4.75-10)(94)$ & $p=0.001$ \\
\hline Theophylline (n) & 146 of $375(37.9)$ & 40 of $95(42.1 \%)$ & 52 of $97(53.6 \%)$ & 25 of $77(32.5 \%)$ & 29 of $106(27.4 \%)$ & $\mathrm{p}=0.001$ \\
\hline Nebuliser use (\%) & 165 of $376(43.9)$ & 45 of $94(47.9)$ & 64 of $97(66.0)$ & 19 of $79(24.0)$ & 37 of $106(34.9)$ & $\mathrm{p}<0.001$ \\
\hline \multicolumn{7}{|l|}{ Steroid-sparing meds } \\
\hline Anti-lgE treatment & 3 of $378(0.8)$ & 0 & 0 & 0 & 3 & $\mathrm{p}=0.054$ \\
\hline PPI (\%) & 111 of $360(29.7)$ & 28 of $95(29.5)$ & 32 of $97(32.9)$ & 18 of $79(22.8)$ & 33 of $107(30.8)$ & $\mathrm{p}=0.498$ \\
\hline Aspirin/NSAID sensitivity (\%) & 36 of $378(9.5)$ & 8 of $93(8.6)$ & 8 of $92(8.7)$ & 10 of $76(13.2)$ & 10 of $105(9.5)$ & $\mathrm{p}=0.741$ \\
\hline Antihistamine (\%) & 89 of $374(23.8)$ & 17 of $94(18.1)$ & 26 of $96(27.1)$ & 16 of $78(20.5)$ & 30 of $106(28.3)$ & $p=0.271$ \\
\hline Nasal steroids (\%) & 96 of $374(25.7)$ & 27 of $94(28.7)$ & 24 of $96(25.0)$ & 17 of $78(21.8)$ & 28 of $106(26.4)$ & $p=0.77$ \\
\hline $\begin{array}{l}\text { Leukotriene receptor } \\
\text { antagonists (\%) }\end{array}$ & 141 of $375(37.6)$ & 52 of $94(55.3)$ & 42 of $96(43.8)$ & 25 of $79(31.6)$ & 22 of $106(20.8)$ & $\mathrm{P}<0.001$ \\
\hline
\end{tabular}
antagonists (\%)

Group data (mean \pm SD or median (IQR) unless stated otherwise) for all subjects are presented in column 2 followed by data for individual centres. Between-centre comparisons for continuous variables were made using one-way analysis of variance or Kruksal-Wallis test (for posthoc comparisons, see text) and for categorical variables using $\chi^{2}$ exact testing.

Significance was taken as $\mathrm{p}<0.01$.

BDP, beclomethasone dipropronate; ICU, intensive care unit; IgE, immunoglobulin E; NSAID, non-steroidal anti-inflammatory drug; PPI, proton pump inhibitor; SABA, short-acting $\beta$-agonist.

cohort. Median body mass index (BMI) was in the non-obese range, and similar to that seen in TENOR (average BMI was 29.9), ${ }^{9}$ whereas the average BMI in ENFUMOSA was lower (male average 26.5, female average 27.2). Current smoking is infrequent, though $29.8 \%$ had previously been smokers, with no difference between centres; these smoking prevalence figures are similar to

Table 5 Lung function

\begin{tabular}{|c|c|c|c|c|c|c|}
\hline & All (382) & Belfast (95) & Brompton (99) & Leicester (79) & Manchester (109) & \\
\hline \multirow{3}{*}{$\%$ predicted } & (371) & (95) & (96) & (77) & $(103)$ & \\
\hline & $1.94 \pm 0.81$ & $1.92 \pm 0.70$ & $1.86 \pm 0.078$ & $2.21 \pm 0.86$ & $1.84 \pm 0.87$ & $\mathrm{p}=0.013$ \\
\hline & $65.9 \pm 23.6$ & $65.7 \pm 24.0$ & $60.4 \pm 20.4$ & $75.3 \pm 22.8$ & $64.7 \pm 25.0$ & $\mathrm{p}=0.001$ \\
\hline \multirow[t]{2}{*}{ FVC (litres) \% predicted } & $3.07 \pm 1.01$ & $3.07 \pm 0.87$ & $3.26 \pm 1.07$ & $3.11 \pm 1.10$ & $2.90 \pm 1.00$ & $p=0.081$ \\
\hline & $81.9 \pm 19.8$ & $82.5 \pm 20.2$ & $82.4 \pm 20.4$ & $83.1 \pm 21.1$ & $79.1 \pm 19.6$ & $p=0.424$ \\
\hline $\mathrm{FEV}_{1} / \mathrm{FVC}$ ratio $\%$ & $63.1 \pm 15.2$ & $62.7 \pm 14.8$ & $56.2 \pm 14.5$ & $71.1 \pm 13.3$ & $63.4 \pm 14.8$ & $\mathrm{p}<0.001$ \\
\hline $\begin{array}{l}\text { Subjects with baseline postbronchodilator } \\
\text { study (n) }\end{array}$ & $(261)$ & $(62)$ & (43) & (75) & $(81)$ & \\
\hline \multirow[t]{2}{*}{ Prebronchodilator $\mathrm{FEV}_{1}$ (litres) (\% predicted) } & $1.90 \pm 0.83$ & $1.69 \pm 0.63$ & $1.83 \pm 0.80$ & $2.22 \pm 0.87$ & $1.81 \pm 0.87$ & $p=0.001$ \\
\hline & $(64.0 \pm 23.1)$ & $(56.9 \pm 18.6)$ & $(58.6 \pm 20.5)$ & $(75.4 \pm 23.0)$ & $(62.7 \pm 24.3)$ & $\mathrm{p}<0.001$ \\
\hline \multirow[t]{3}{*}{ Prebronchodilator FVC (litres) (\% predicted) } & $3.05 \pm 1.05$ & $2.99 \pm 0.94$ & $3.36 \pm 1.15$ & $3.11 \pm 1.10$ & $2.89 \pm 1.02$ & $\mathrm{p}=0.128$ \\
\hline & & & & & & $p=0.220$ \\
\hline & $(80.6 \pm 19.8)$ & $(79.0 \pm 19.3)$ & $(84.1 \pm 18.1)$ & $(83.3 \pm 21.4)$ & $(77.8 \pm 19.3)$ & \\
\hline \multirow[t]{3}{*}{ Postbronchodilator FEV 1 (litres) (\% predicted) } & & & & & & $p=0.020$ \\
\hline & $2.18 \pm 0.88$ & $1.93 \pm 0.70$ & $2.29 \pm 1.03$ & $2.36 \pm 0.86$ & $2.14 \pm 0.89$ & $p=0.004$ \\
\hline & $(73.6 \pm 24.2)$ & $(65.3 \pm 21.4)$ & $(73.6 \pm 26.3)$ & $(80.7 \pm 22.5)$ & $(74.2 \pm 24.7)$ & \\
\hline \multirow[t]{3}{*}{ Postbronchodilator FVC (litres) (\% predicted) } & & & & & & $p=0.246$ \\
\hline & $3.29 \pm 1.06$ & $3.21 \pm 0.97$ & $3.60 \pm 1.26$ & $3.30 \pm 1.11$ & $3.23 \pm 0.95$ & $p=0.664$ \\
\hline & $(87.4 \pm 19.1)$ & $(85.2 \pm 19.9)$ & $(90.9 \pm 21.1)$ & $(87.8 \pm 20.3)$ & $(87.4 \pm 16.4)$ & \\
\hline \multirow[t]{2}{*}{ Postbronchodilator $\mathrm{FEV}_{1} / \mathrm{FVC}$ ratio (\%) } & & & & & & $\mathrm{p}<0.001$ \\
\hline & $65.0 \pm 14.6$ & $60.6 \pm 12.4$ & $61.44 \pm 15.82$ & $71.6 \pm 12.3$ & $65.0 \pm 15.6$ & \\
\hline Total lung capacity \% predicted (n) & $104.9 \pm 16.8(265)$ & $107.8 \pm 17.8(80)$ & $109.1 \pm 13.9(96)$ & & $98.0 \pm 16.6(89)$ & $\mathrm{p}<0.001$ \\
\hline Residual volume \% predicted (n) & $134.4 \pm 42.0(263)$ & $127.8 \pm 44.2(80)$ & $151.0 \pm 40.1(97)$ & & $121.8 \pm 35.9(86)$ & $\mathrm{p}<0.001$ \\
\hline $\mathrm{K}_{\mathrm{CO}} \%$ predicted $(\mathrm{n})$ & $101.5 \pm 17.5(256)$ & $97.3 \pm 17.7(79)$ & $98.0 \pm 13.8(94)$ & & $110.04 \pm 18.5(83)$ & $\mathrm{p}<0.001$ \\
\hline
\end{tabular}

Group data (mean \pm SD) for all subjects are presented in column 2 followed by data for individual centres. Between-centre comparisons for continuous variables were made using one-way analysis of variance (for posthoc comparisons, see text).

Significance was taken as $\mathrm{p}<0.01$.

$\mathrm{FEV}_{1}$, forced expiratory volume in $1 \mathrm{~s}$. FVC, forced vital capacity, $\mathrm{K}_{\mathrm{CO}}$, carbon monoxide transfer coefficient. 
Table 6 Blood testing and bone density

\begin{tabular}{|c|c|c|c|c|c|c|}
\hline & All (382) & Belfast (95) & Brompton (99) & Leicester (79) & Manchester ('109) & \\
\hline Blood eosinophil count $\times 10^{9} / \mathrm{l}(\mathrm{n})$ & $\begin{array}{l}0.30 \\
(0.25-11.0) \\
(360)\end{array}$ & $\begin{array}{l}0.34 \\
(0.19-0.72) \\
(94)\end{array}$ & $\begin{array}{l}0.1 \\
(0.2-0.5) \\
(98)\end{array}$ & $\begin{array}{l}0.12 \\
(0.35-0.58) \\
(72)\end{array}$ & $\begin{array}{l}0.24 \\
(0.08-0.54) \\
(96)\end{array}$ & $p=0.001$ \\
\hline FeNO ppb (n) & $\begin{array}{l}34.5 \\
(16-65) \\
(133)\end{array}$ & $\begin{array}{l}40 \\
(17-92) \\
(57)\end{array}$ & $\begin{array}{l}30.5 \\
(14-65) \\
(24)\end{array}$ & $\begin{array}{l}34 \\
(12-60) \\
(52)\end{array}$ & & $p=0.321$ \\
\hline $\lg \mathrm{E} \mathrm{kU} / \mathrm{l}(\mathrm{n})$ & $\begin{array}{l}130 \\
(53.5-292) \\
(349)\end{array}$ & $\begin{array}{l}113 \\
(60-256) \\
(93)\end{array}$ & $\begin{array}{l}166.5 \\
(51.5-299.5) \\
(96)\end{array}$ & $\begin{array}{l}126 \\
(50-335.5) \\
(77)\end{array}$ & $\begin{array}{l}140 \\
(51-280) \\
(83)\end{array}$ & $p=0.831$ \\
\hline $\lg G \mathrm{~g} / \mathrm{l}(\mathrm{n})$ & $\begin{array}{l}9.3 \\
(7.85-10.9) \\
(195)\end{array}$ & $\begin{array}{l}9.15 \\
(7.9-10.0) \\
(86)\end{array}$ & $\begin{array}{l}9.1 \\
(7.0-10.0) \\
(63)\end{array}$ & $\begin{array}{l}9.85 \\
(8.1-11.77) \\
(46)\end{array}$ & & $p=0.052$ \\
\hline $\lg M g / l(n)$ & $\begin{array}{l}1.0 \\
(0.8-1.4) \\
(195)\end{array}$ & $\begin{array}{l}1.0 \\
(0.7-1.4) \\
(86)\end{array}$ & $\begin{array}{l}1.1 \\
(0.88-1.4) \\
(63)\end{array}$ & $\begin{array}{l}1.1 \\
(0.72-1.42) \\
(46)\end{array}$ & & $p=0.588$ \\
\hline Bone density (T scores) ${ }^{*}(\mathrm{n})$ & (169) & (41) & (70) & & (58) & \\
\hline $\mathrm{L} 1-\mathrm{L} 4$ & $-0.64 \pm 1.42$ & $-0.81 \pm 1.15$ & $-0.54 \pm 1.34$ & & $-0.67 \pm 1.67$ & $p=0.603$ \\
\hline Femoral neck & $-0.26 \pm 1.20$ & $-0.22 \pm 0.84$ & $-0.07 \pm 1.28$ & & $-0.61 \pm 1.19$ & $p=0.017$ \\
\hline
\end{tabular}

${ }^{*} 18(10.6 \%)$ subjects had either a spinal or femoral neck bone density less than or equal to -2.5 (osteoporosis) and 58 (34.3\%) subjects had a spinal or femoral neck bone density less than or equal to -1.0 (osteopenia).

Group data (mean \pm SD or median (range)) for all subjects are presented in column 2 followed by data for individual centres. Between-centre comparisons for continuous variables were made using one-way aanalysis of variance or Kruksal-Wallis test (for posthoc comparisons, see text) and for categorical variables using $\chi^{2}$ analysis.

Significance was taken as $\mathrm{p}<0.01$.

FeNO, fractional exhaled nitric oxide; Ig, immunoglobulin.

the TENOR cohort where $4.3 \%$ of 'difficult to control' adults were current smokers, $32 \%$ ex-smokers and $63.7 \%$ never smokers. ${ }^{9}$

In Leicester, more patients were in full-time employment, compared with $33-36 \%$ in other centres, but why asthma is less often volunteered as the reason for not working in Belfast remains unclear, though this would significantly impact on costing refractory asthma. Occupational asthma worsening was consistently uncommon, and only appears relevant to a minority of patients.

Three centres recorded a family history of asthma in $42-44 \%$, but in Belfast this was $74 \%$. Retrospective data checking

Table 7 General linear models for listed dependent variables: dependent variables were entered as the square root of the index variable to ensure residuals in the model were normally distributed

\begin{tabular}{|c|c|c|c|}
\hline Dependent variable & Factors/co-variates & Regression coefficients (95\% CI) & p Value \\
\hline \multirow{7}{*}{$\begin{array}{l}\text { Hospital admission in } 12 \text { months prior to } \\
\text { clinical assessment }\end{array}$} & Unscheduled visits in prior 12 months & $0.051(0.033$ to 0.071$)$ & $\mathrm{p}<0.001$ \\
\hline & $\begin{array}{l}\text { Total number of prior intensive care } \\
\text { admissions }\end{array}$ & $0.086(0.012$ to 0.16$)$ & $\mathrm{p}<0.05$ \\
\hline & Hospital centre & Belfast $-0.108(-0.341$ to 0.126$)$ & $\mathrm{p}<0.001$ \\
\hline & & Brompton 0.418 (0.187 to 0.650$)$ & \\
\hline & & Leicester $0.077(-0.160$ to 0.314$)$ & \\
\hline & & Manchester 0 & \\
\hline & On theophylline at initial assessment & $0.192(0.017$ to 0.366$)$ & $\mathrm{p}<0.05$ \\
\hline \multirow{7}{*}{$\begin{array}{l}\text { Rescue steroids in } 12 \text { months prior to } \\
\text { clinical assessment }\end{array}$} & Hospital centre & Belfast 0.588 (0.295 to 0.881$)$ & $\mathrm{p}<0.001$ \\
\hline & & Brompton 0.784 (0.490 to 1.078$)$ & \\
\hline & & Leicester 0.757 (0.458 to 1.055$)$ & \\
\hline & & Manchester 0 & \\
\hline & Blood eosinophils at first assessment & $0.238(0.105$ to 0.372$)$ & $\mathrm{p}<0.001$ \\
\hline & $\begin{array}{l}\text { Age at first assessment at Difficult } \\
\text { Asthma Service }\end{array}$ & $-0.001(-0.019$ to -0.003$)$ & $\mathrm{p}<0.01$ \\
\hline & Nebuliser usage at initial assessment & $0.223(0.004$ to 0.443$)$ & $\mathrm{p}<0.05$ \\
\hline \multirow[t]{7}{*}{$\begin{array}{l}\text { Unscheduled visits in } 12 \text { months prior to } \\
\text { clinical assessment }\end{array}$} & $\begin{array}{l}\text { Age at first assessment at difficult asthma } \\
\text { service }\end{array}$ & $-0.011(-0.019$ to -0.003$)$ & $\mathrm{p}<0.01$ \\
\hline & Hospital centre & Belfast $0.500(0.188$ to 0.813$)$ & $\mathrm{p}<0.01$ \\
\hline & & Brompton $0.148(-0.159$ to 0.454$)$ & \\
\hline & & Leicester $0.418(-0.090$ to 0.746$)$ & \\
\hline & & Manchester 0 & \\
\hline & Gender & $0.284(0.051$ to 0.518$)$ & $\mathrm{p}<0.05$ \\
\hline & Blood eosinophils at first assessment & $0.147(0.003$ to 0.290$)$ & $\mathrm{p}<0.05$ \\
\hline
\end{tabular}


confirmed that the 'family history' definition was applied consistently, and a family history appears more common in Northern Ireland, which has traditionally low levels of immigration. Recorded familial asthma death was low, suggesting it is an infrequent event, even in this refractory group; however, this was not well recorded and we cannot exclude that the familial asthma death rate may be higher.

This higher reported atopic prevalence in London was supported by allergen testing (table 3 ), as subjects were more likely to be allergen positive. The difference is particularly notable between London and Belfast, where all subjects were tested for similar allergens. In Leicester and Manchester, targeted allergen testing is performed, and reported allergen positivity may represent an underestimation. Interestingly, Apergillus sensitivity in Manchester (which is routinely tested) was 'between' that of London and Belfast. In SARP, 71\% of those with severe asthma were skin prick positive to $\geq 1$ of 14 allergens $(85 \%$ and $87 \%$ for subjects with mild/moderate asthma, respectively). ${ }^{11}$ In ENFUMOSA, $\sim 58 \%$ of those with severe asthma had positivity $\geq 1$ allergen, (well controlled asthmatics $\sim 76 \%$ ), with individual allergen positivity in those with severe asthma varying between $\sim 10 \%$ and $35 \% .{ }^{10}$ Individual centre data are not presented in these studies; however, our data suggest that individual allergen sensitisation appears to vary in different refractory asthma populations in UK specialist centres, possibly reflecting important regional differences even within the UK.

Oral steroid courses in the 12 months before referral were fewer in Manchester compared with other centres, where the median number of rescue steroids was 4-5 per annum; this difference may reflect patient recollection in Manchester, but the similarity in other centres supports significant rescue steroid use in this group. In SARP, $54 \%$ of those with severe asthma reported $\geq 3$ steroid bursts per year ${ }^{11}$ and, in our cohort, $63 \%$ used $\geq 3$ courses in the previous year. Unscheduled visits were more common in Belfast, although hospital admissions and ICU usage were higher in London, though they were uncommon events. In SARP, $54 \%$ reported $\geq 1$ unscheduled care visit per year, ${ }^{11}$ whereas this was $86 \%$ for this UK cohort. It is unclear if differences are due to different healthcare delivery for exacerbation management, or to differences in exacerbation severity.

Almost twice as many patients in the Brompton were on maintenance steroids, compared with the other centres. Inhaled steroid dose at referral was also higher in the Brompton patients compared with all other centres. The UK cohort average inhaled steroid dose was similar to the ENFUMOSA severe asthma cohort (1676 $\pm 667 \mu \mathrm{g}$ BDP equivalent), but comparative data between centres were not presented. Our data suggest that in multicentre studies specifying a minimum steroid dose for inclusion, it is important to examine medication utilisation from individual centres.

Wide variation was noted in nebuliser usage, and patients in Belfast and Brompton were more likely to be on a theophylline and leukotriene receptor antagonist at referral. These differences presumably relate to local prescribing practice and referral pattern, but all patients were on multiple medications at referral. Few patients were on steroid-sparing medication or antiIgE treatment at referral, suggesting that these medications are not widely used outside specialist centres.

We observed a low prevalence of aspirin/non-steroidal antiinflammatory drug (NSAID) sensitivity and, while different definitions of aspirin sensitivity probably affect reported prevalence, ${ }^{12}$ our data are notably different from those of ENFUMOSA, which suggested an association between asthma severity and self-reported aspirin exacerbation. ${ }^{10}$ Our data are similarly based on self-reported increased asthma symptoms after aspirin/NSAID ingestion, and the difference may reflect differences between the UK and a European population.

Spirometry for the UK group was lower than for the ENFUMOSA study ( $\mathrm{FEV}_{1} \%$ predicted $71.8 \%$ rising to $80.9 \%$ postbronchodilator), though it was similar to SARP (FEV $1 \%$ predicted $62 \pm 22 \%$ ), consistent with a patient population with more severe asthma. Consistent with better spirometry in ENFUMOSA, total lung capacity in the UK cohort was higher

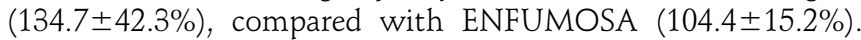
Of note, $\mathrm{K}_{\mathrm{CO}}$ was normal and similar in all centres despite the high prevalence of ex-smoking in the UK cohort, though we demonstrated a relationship between $\mathrm{K}_{\mathrm{CO}}$ and prior smoking. The normal percentage $\mathrm{K}_{\mathrm{CO}}$ is again different from ENFUMOSA, where $\mathrm{K}_{\mathrm{CO}}$ was $90.6 \pm 19 \%$ predicted; the authors suggested that abnormal gas exchange and parenchymal injury may be a feature of severe asthma. However, our data suggest that in a well characterised population with refractory asthma, the transfer factor is well preserved, and any reductions relate to prior smoking history.

Interesting differences between UK centres are seen for spirometry. Prebronchodilator and postbronchodilator spirometry and degree of airflow obstruction were better in Leicester compared with other centres. Allied with other indices-for example, increased numbers in full-time work-it suggests less severe disease in the Leicester cohort compared with other centres. This is despite the fact that all of these patients fulfilled the same definition of refractory asthma, suggesting a spectrum of severity using the ATS definition. Prospective analysis of patient outcome and treatment requirements, which is facilitated by the UK Registry, will help address this issue further Residual volume \% predicted and total lung capacity were significantly greater in the London cohort compared with Belfast and Manchester; however, the absolute differences are small.

Peripheral blood eosinophil count was highest in Belfast, but there was no significant difference in sputum eosinophilia or FeNO between centres. In SARP, FeNO data were available on 135 subjects with severe asthma and FeNO was $40 \pm 38 \mathrm{ppb}$, which is similar to this UK cohort. Mean IgE in ENFUMOSA was 109 (95\% CI 85 to 139), and in SARP 100土5.75. We have presented IgE as median (IOR) as this is not normally distributed in this cohort, but our median value of 130 is comparable with other published cohorts. In subjects who had bone density measurements, 18 (10.6\%) subjects had either a spinal or femoral neck bone density $\mathrm{T}$ score less than or equal to -2.5 (osteoporosis) and 58 (34.3\%) subjects had a T score less than or equal to -1.0 (osteopenia). Bone density measurements were not done in all subjects and there may be some selection bias; however, these data are consistent with a significant morbidity in this group.

Important clinical outcomes in this group are unscheduled healthcare visits, rescue steroid use, hospital admission and ICU admission. Hierarchical regression modelling supported important between-centre differences, as this remained a significant factor in the final model for three of the four tested dependent variables (table 7). It is important to note that if this patient group was treated as a homogeneous study population, different regression models were obtained, with other additional significant associations, which were not demonstrated when centre was included as a factor (data not shown). Importantly, however, the factors which remained in our regression models with clinical centre included as a random factor also remained in each regression model, independent of whether centre was included or not. 
The association between peripheral blood eosinophils and both steroid exposure and unscheduled visits in this refractory patient group is consistent with other studies which have demonstrated a relationship between blood and/or sputum eosinophilia and asthma exacerbation and loss of asthma control. ${ }^{13-15}$ Patients who were younger at the time of assessment at the Difficult Asthma Service had higher levels of both unscheduled visits and hospital admissions in the respective models. The association between total number of prior ICU admissions and hospital admission is perhaps not surprising as prior severe asthmatic events are associated with increased risk of asthma death. ${ }^{16}{ }^{17}$ Home nebuliser usage and theophylline prescription may also be markers of severity and thus related to increased rescue steroid exposure and unscheduled visits, respectively. The association with female gender and unscheduled visits is interesting, particularly as this gender effect is not translated into either more steroid exposure or hospital admission, and might suggest a lower threshold for healthcare contact in females. The association between dose of inhaled steroids and ICU admission is interesting, particularly as this has previously been shown to be a predictor of treatment-resistant asthma ${ }^{5}$ and is presumably an index of longer term severity with increasing doses of inhaled steroid over time. The association with age of asthma diagnosis may reflect ICU admission in childhood but, even if this is the case, it does suggest that severe asthma in this group is present from an early stage after the initial diagnosis.

In summary, this paper presents for the first time demographic, physiological and immunological data from a well characterised UK population of subjects with refractory asthma. While similarities exist to other comparable published cohorts, there are also differences which may reflect different patient populations (difficult to control vs refractory vs different levels of asthma severity), and again argues for the production and application of precise definitions for this population of subjects with asthma. In addition, important population characteristics are different between UK specialist centres, suggesting that 'grouping' multicentre data (even centres in the same country) may miss important differences and potentially confound attempts to phenotype refractory asthma, giving rise to so-called 'ecological fallacy'. The differences identified between UK centres warrant further study, but suggest refractory asthma may be more heterogeneous within an individual country than previously known.

Acknowledgements We thank the data input staff and medical and nursing staff in the UK Difficult Asthma Centres-Belfast, Jacqui Gamble and Kirsty Graham;
Leicester, Sarah Terry, Amisha Singapuri, Beverley Hargadon, Maria Shelley, Ruth Green, lan Pavord, Andrew Wardlaw and Peter Bradding; London, Debbie Campbell and Suzanne Regan; Manchester, Gill McCumesky and Leanne Holmes.

Funding Pilot funding for the Registry was provided as unrestricted research grants from Astra Zeneca, Glaxo Smith Kline and Novartis. CB is supported by a Wellcome Senior Clinical Fellowship.

\section{Competing interests None.}

Contributors LGH is Co-ordinator of the British Thoracic Society UK Difficult Asthma Registry and with CEB, AM-G and RMN co-lead the British Thoracic Society Difficult Asthma Network, and all have contributed equally to this manuscript.

Provenance and peer review Not commissioned; externally peer reviewed.

\section{REFERENCES}

1. British Thoracic Society Scottish Intercollegiate Guidelines Network. British guideline on the management of asthma. Thorax 2008;63(Suppl 4):iv1-121.

2. Smith DH, Malone DC, Lawson KA, et al. A national estimate of the economic costs of asthma. Am J Respir Crit Care Med 1997;156:787-93

3. Barnes PJ, Jonsson B, Klim JB. The costs of asthma. Eur Respir J 1996;9:636-42.

4. Chanez P, Wenzel SE, Anderson GP, et al. Severe asthma in adults: what are the important questions? J Allergy Clin Immunol 2007;119:1337-48.

5. Heaney LG, Conway E, Kelly C, et al. Predictors of therapy resistant asthma: outcome of a systematic evaluation protocol. Thorax 2003:58:561-6.

6. Robinson DS, Campbell DA, Durham SR, et al. Asthma and Allergy Research Group of the National Heart and Lung Institute. Systematic assessment of difficult-to-treat asthma. Eur Respir J 2003;22:478-83.

7. Araujo AC, Ferraz E, Borges Mde C, et al. Investigation of factors associated with difficult-to-control asthma. J Bras Pneumol 2007;33:495-501.

8. Anon. Proceedings of the ATS workshop on refractory asthma: current understanding, recommendations, and unanswered questions. American Thoracic Society. Am J Respir Crit Care Med 2000;162:2341-51.

9. Dolan CM, Fraher KE, Bleecker ER, et al. TENOR Study Group. Design and baseline characteristics of the epidemiology and natural history of asthma: Outcomes and Treatment Regimens (TENOR) study: a large cohort of patients with severe or difficult-to-treat asthma. Ann Allergy Asthma Immunol 2004;92:32-9.

10. Anon. The ENFUMOSA cross-sectional European multicentre study of the clinical phenotype of chronic severe asthma. European Network for Understanding Mechanisms of Severe Asthma. Eur Respir J 2003;22:470-7.

11. Moore WC, Bleecker ER, Curran-Everett D, et al; for the National Heart, Lung, and Blood Institute's Severe Asthma Research Program. Characterization of the severe asthma phenotype by the National Heart, Lung, and Blood Institute's Severe Asthma Research Program. J Allergy Clin Immunol 2007;119:405-13.

12. Szczeklik A, Nizankowska E, Duplaga M, et al; on behalf of the AIANE investigators Natural history of aspirin-induced asthma. Eur Respir J 2000;16:432-6.

13. Belda J, Parameswaran $\mathrm{K}$, Lemière $\mathrm{C}$, et al. Predictors of loss of asthma control induced by corticosteroid withdrawal. Can Respir J 2006;13:129-33.

14. Jatakanon A, Lim S, Barnes PJ. Changes in sputum eosinophils predict loss of asthma control. Am J Respir Crit Care Med 2000;161:64-72.

15. Green R, Brightling C, McKenna S, et al. Asthma exacerbations and sputum eosinophil counts: a randomised controlled trial. Lancet 2002;360:1715-21.

16. Jalaludin BB, Smith MA, Chey T, et al. Risk factors for asthma deaths: a populationbased, case-control study. Aust NZ J Public Health 1999;23:595-600.

17. Rea HH, Scragg R, Jackson R, et al. A case-control study of deaths from asthma. Thorax 1986;41:833-9. 